\title{
Selective serotonin reuptake inhibitor treatment
} in the UK: risk of relapse or recurrence

\section{of depression}

\author{
AMI J. CLAXTON, ZHIMING LI and JAN MCKENDRICK
}

\author{
Background Patients with depression \\ are often not prescribed antidepressants \\ for an adequate period of time.
}

\begin{abstract}
Aims The impact of antidepressant prescribing patterns on the risk of relapse or recurrence of depression is examined.
\end{abstract}

Method The MediPlus UK Primary Care Database was used to identify patients treated for depression with a selective serotonin reuptake inhibitor (SSRI). Records were used to construct hierarchical prescription patterns (less than 120 days, switching/augmentation, upward titration, or stable use) as indicators for the occurrence of relapse or recurrence of depression.

Results Patients with stable use experienced the lowest risk of relapse or recurrence. Factors significantly associated with increased risk include prior use of anxiolytic medications, more comorbid conditions and younger age.

Conclusions The SSRI prescription pattern most consistent with recommended depression treatment guidelines was associated with the lowest risk of relapse or recurrence.

Declaration of interest Funding provided by Eli Lilly and Company.
Depression is often a chronic and recurrent disorder (Kocsis et al, 1996). Current guidelines for the treatment of depression (Paykel \& Priest, 1992; Depression Guideline Panel, 1993; Montgomery et al, 1993) recommend 4-9 months of antidepressant treatment after acute symptom resolution to prevent relapse (re-emergence of original symptoms) and recurrence (a new episode) of depression. Previous clinical studies have demonstrated that long-term therapy significantly reduces the risk of relapse and recurrence (Frank et al, 1990; Maj et al, 1992; Old Age Depression Interest Group, 1993; Prien \& Kupfer, 1986). Furthermore, in a recent analysis of Medicaid claims of patients with depression in the USA (Melfi et al, 1998), antidepressant prescribing patterns were constructed as proxy measures of guideline adherence, and a utilisation-based definition of relapse or recurrence of depression was defined. The study reported that patients who prematurely discontinued antidepressant treatment had a significantly increased risk of relapse or recurrence of depression, while those who continued therapy were least likely to experience relapse or recurrence of depression.

Here, the same methodology is utilised in a primary-care setting in the UK to explore further the effects of prescribing patterns in clinical practice on relapse and recurrence of depression. The focus here is on prescribing patterns within one class of antidepressants, the selective serotonin reuptake inhibitors (SSRIs), in order to allow for better characterisation of the impact of the treatment pattern itself and because of their increasing first-line use.

\section{METHOD}

Study periods and sample selection

All data used in this analysis came from the MediPlus UK Primary Care Database, a general practice database containing anonymised, computerised clinical records for almost two million patients in the UK (approximately one million currently active). The database contains complete general practice records on diagnoses, symptoms, prescriptions, hospital/specialist referrals, tests and administrative notes.

From the MediPlus database, the patients initially extracted were those active in the database on 30 November 1997 and who were prescribed any antidepressant in the index window from 1 January 1993 to 30 November 1995 ( $n=32$ 496). The index window was chosen to ensure adequate duration of both pre-study screening and follow-up of each patient, given the availability of data. The patient's first antidepressant prescription in the index window is referred to as the index drug; the prescription date of the index drug is referred to as the index date. For each patient, three non-overlapping study periods were identified in reference to their index date: the prior period $(6$ months before the index date); the treatment period (6 months after the index date); and the follow-up period (18 months after the end of the treatment period).

From the initial patient extraction, all adult (age 18 years or more) patients who were prescribed one of the three most commonly prescribed SSRI antidepressants (fluoxetine, paroxetine and sertraline; $n=10308$ ) in the UK were included. Of these patients, only those who had evidence of a depression diagnosis within 30 days of the index date (before or after), as determined by the Read clinical classification codes (Chisholm, 1990) (Table 1), were included $(n=8704)$.

To maximise the likelihood of capturing 'new' episodes of depression in the sample, patients were required to have no evidence of either a diagnosis of depression or treatment with any antidepressant during a 6-month prior period $(n=7739)$. Furthermore, to ensure that patients were truly free of any antidepressant use in this period, only patients who had at least one resource use during the 2 years before the index prescription were included $(n=7542)$. This procedure distinguishes patients whose lack of evidence of any antidepressant use was due to non-eligibility for services (and who may have obtained an antidepressant from another source) from patients who were eligible but did not receive a prescription for any antidepressant. Lastly, patients with evidence 
Table I Read codes' used for identification of patients with depression

\begin{tabular}{lll}
\hline Read code & Depression type & $\begin{array}{l}\text { ICD-9 - clinical } \\
\text { modification }{ }^{2} \text { equivalent }\end{array}$ \\
\hline IBI7 & Depressed & $296.20-23,296.25-26$ \\
2257 & On examination depressed & $296.30-33,296.35-36$ \\
E222 & Endogenous depression & 300.4 \\
E35 & Reactive (neurotic) depression & $309.0,309.1$ \\
E4J5 & Depression not otherwise specified & 311 \\
\hline
\end{tabular}

I. Read clinical classification code

2. Copyright 1986: Commission on Professional and Hospital Activities, 1968 Green Road, Ann Arbor, Ml 48I05, USA.

of other comorbid psychiatric conditions (dementia, schizophrenia, psychosis, manic depression) during the prior period and/or the follow-up period were excluded, leaving a final sample of 7293 patients.

\section{Definition of relapse and recurrence of depression}

The dependent variable in this study was the presence of a relapse or recurrence during the 18-month follow-up period, as defined from the clinical record. Because neither relapse nor recurrence of depression was systemically noted in this database, a proxy measure of relapse or recurrence of depression was defined as one or more of the following events occurring during the follow-up period: (a) re-initiation of any antidepressant treatment after a gap of at least 6 months with no antidepressant prescription; (b) suicide attempt, referral to psychotherapy or psychiatrist, admission to a mental health facility, emergency room use related to mental disorders, or electroconvulsive therapy; (c) re-initiation and an event listed under (b). Based on these criteria, variables indicating the presence and type of relapse or recurrence of depression for each individual in the sample were constructed.

\section{Prescribing patterns}

From the antidepressant prescription records during the treatment period, four patient cohorts of mutually exclusive prescribing patterns were defined hierarchically from prescription records which included drug, dose and duration of the prescription. The total number of antidepressant days was used to determine length of treatment during the treatment period. From these records, the $<120$ days cohort was defined as those patients who had fewer than 120 days of any antidepressant treatment during the 6 months following their index prescription. This corresponds to a minimum period of 60 or more days without antidepressant prescription coverage (continuous or divided) during the treatment period. Next, the switching/augmentation cohort was defined as patients who had at least 120 days of therapy, but who switched agents or added a second antidepressant (SSRI or other antidepressant). A third group of patients, identified as the upward titration cohort, maintained treatment on the original SSRI for at least 120 days, but had their dosage increased at some point during the 6-month treatment period. Finally, all remaining patients in the sample were the stable use cohort, having at least 120 days of SSRI therapy on the index SSRI at a stable dosage level throughout the treatment period. In all analyses, the stable use cohort served as the reference category with which other cohorts were compared.

\section{Other explanatory variables}

Other variables potentially related to relapse or recurrence and/or prescribing patterns were considered. These included baseline demographic characteristics (age, gender, marital status and geographical region based on former health authority regions). The year of the index prescription was also included in order to account for possible differences in prescribing patterns during different calendar years. A binary variable representing the use of an axiolytic agent during the prior period was constructed. The effects of non-psychiatric comorbid conditions on relapse or recurrence of depression were measured by a simple count of non-psychiatric diagnoses noted in the prior period. Unique diagnoses were categorised according to ICD-9 codes (World Health Organization, 1978) at the three-digit level and then summed.

\section{Statistical methods}

Data analysis was carried out using SAS for Windows, release 6.12 (SAS Inc., Cary, NC). Descriptive statistics for the total sample, stratified by the outcome, were calculated. The effect of prescribing patterns observed during the first 6 months of treatment on the occurrence of relapse or recurrence during the subsequent 18 months was first estimated by Kaplan-Meier curves. Logistic regression was then used to estimate the impact of prescribing patterns on relapse or recurrence while adjusting for other explanatory variables. Results from the logistic regression model are presented as adjusted odds ratios (ORs) with the corresponding $95 \%$ confidence intervals (CIs).

\section{RESULTS}

A total of 7493 patients were included in the analysis (Table 2). The mean age was 43 years (s.d. $=16$ years) and the majority of patients were female $(72 \%)$. Patients had an average of 2.2 non-psychiatric diagnoses during the 6 months prior to initiation with an SSRI antidepressant. Only a small percentage $(1.2 \%)$ of patients were prescribed anxiolytics during the prior period.

On average, patients had 2.9 antidepressant prescriptions with a mean antidepressant supply of 85 days during the 6-month treatment period. The majority of patients in the sample $(73 \%)$ had fewer than 120 days of antidepressant therapy (mean 54.2 days, s.d. $=32.3$ ) in the first 6 months of treatment $(<120$ days cohort). Approximately $4 \%$ of patients remained on an antidepressant for at least 120 days (mean 189.7 days, s.d. $=48.8$ ), but switched to or augmented with another antidepressant (switching/augmentation cohort). Only $2 \%$ of patients remained on an antidepressant for at least 120 days (mean 178.9 days, s.d.=34.3) but had their initial dose increased (upward titration cohort). The remainder of the sample $(21 \%)$ maintained therapy on the same drug for more than 120 days (mean 164.2 days, s.d.=29.2) and did not titrate upward (stable use cohort)

Overall, 1691 patients $(22.6 \%)$ met the criteria for a relapse or recurrence of 
depression during the 18-month follow-up period. The mean number of days between the initiation of SSRI therapy (the index date) and the relapse or recurrence was
263 days (s.d.=153 days) for all patients with a relapse or recurrence. Of those patients with a relapse or recurrence, $67.5 \%$ were identified by re-initiation of

Table 2 Descriptive statistics of adult patients prescribed an SSRI for depression (n (\%) unless otherwise shown)

\begin{tabular}{|c|c|c|c|}
\hline & \multirow[t]{2}{*}{ Total' } & \multicolumn{2}{|c|}{ Had relapse/recurrence? } \\
\hline & & Yes & No \\
\hline Total sample & $7493(100 \%)$ & I69I (22.6\%) & $5802(77.4 \%)$ \\
\hline \multicolumn{4}{|l|}{ Prescription pattern } \\
\hline$<120$ days & 5459 (72.8\%) & 1255 (23.0\%) & $4204(77.0 \%)$ \\
\hline Switching/augmentation & $285(3.8 \%)$ & 83 (29.1\%) & $202(70.9 \%)$ \\
\hline Upward titration & $148(2.0 \%)$ & $35(23.6 \%)$ & II3 (76.4\%) \\
\hline Stable use & 1601 (2I.4\%) & $318(19.9 \%)$ & $1283(80.1 \%)$ \\
\hline \multicolumn{4}{|l|}{ Comorbidity } \\
\hline Anxiolytic use in prior period & 87 (I.2\%) & $34(39.1 \%)$ & $53(60.9 \%)$ \\
\hline No anxiolytic use in prior period & $7406(98.8 \%)$ & 1657 (22.4\%) & $5749(77.6 \%)$ \\
\hline Number of prior-period comorbidities (mean (s.d.)) & $2.2(1.6)$ & $2.4(1.7)$ & $2.2(1.6)$ \\
\hline \multicolumn{4}{|l|}{ Index year } \\
\hline 1993 & $2022(27.0 \%)$ & 474 (23.4\%) & $1548(76.6 \%)$ \\
\hline 1994 & $2355(31.4 \%)$ & 551 (23.4\%) & $1804(76.6 \%)$ \\
\hline 1995 & $3116(41.6 \%)$ & $666(21.4 \%)$ & $2450(78.6 \%)$ \\
\hline \multicolumn{4}{|l|}{ Patient characteristics } \\
\hline Age (mean (s.d.)) & $43.3(16.2)$ & $4 I .8(15.7)$ & $43.8(16.4)$ \\
\hline Female & $5413(72.2 \%)$ & $125 \mid$ (23.1\%) & $4162(76.9 \%)$ \\
\hline Male & $2080(27.8 \%)$ & 440 (2I.2\%) & $1640(78.8 \%)$ \\
\hline Cohabiting & $59(0.8 \%)$ & $15(25.4 \%)$ & $44(74.6 \%)$ \\
\hline Divorced & $161(2.1 \%)$ & $38(23.6 \%)$ & $123(76.4 \%)$ \\
\hline Separated & $48(0.6 \%)$ & $12(25.0 \%)$ & $36(75.0 \%)$ \\
\hline Single & 859 (11.5\%) & 224 (26.1\%) & $635(73.9 \%)$ \\
\hline Widowed & $159(2.1 \%)$ & $30(18.9 \%)$ & $129(81.1 \%)$ \\
\hline Married or unknown & 6207 (82.9\%) & 1372 (22.1\%) & 4835 (77.9\%) \\
\hline Region $\left.\right|^{2}$ & $1554(20.7 \%)$ & 335 (21.6\%) & $1219(78.4 \%)$ \\
\hline Region 2 & $1636(21.8 \%)$ & 391 (23.9\%) & 1245 (76.1\%) \\
\hline Region 3 & $2149(28.7 \%)$ & 489 (22.8\%) & 1660 (77.2\%) \\
\hline Region 4 & 2154 (28.8\%) & 476 (22.1\%) & 1678 (77.9\%) \\
\hline
\end{tabular}

SSRI, selective serotonin reuptake inhibitor.

I. For the total column, percentages are of the total sample (7493). For the relapse/recurrence subsample, percentages are of the row total.

2. Region I: East Anglia, Oxford and NorthThames; region 2: South Thames, South Western, Wessex; region 3: West Midlands, Mersey, Trent and Wales; region 4: rest of UK.

Table 3 Type of relapse or recurrence by prescription pattern cohort ( $(\%))$

\begin{tabular}{lcccc}
\hline & $\begin{array}{c}\text { Early discontinuation } \\
n(\%)\end{array}$ & $\begin{array}{c}\text { Switching/augmentation } \\
\text { (1) }\end{array}$ & $n(\%)$ & Upward titration \\
$\begin{array}{l}\text { Total relapse or } \\
\text { recurrence }\end{array}$ & $1313(77.6 \%)$ & $24(1.4 \%)$ & Stable use \\
$\begin{array}{l}\text { Both re-initiation } \\
\text { and event }\end{array}$ & $172(81.1 \%)$ & $35(2.1 \%)$ & $319(18.9 \%)$ \\
$\begin{array}{l}\text { Re-initiation only } \\
\text { Event only }\end{array}$ & $930(81.4 \%)$ & $1(0.5 \%)$ & $7(3.3 \%)$ & $32(15.1 \%)$ \\
\hline
\end{tabular}

$\chi^{2}$ test of independence, $P<0.00$ I, d.f. $=4$.

therapy with any antidepressant, $19.9 \%$ by re-initiation of an antidepressant and experiencing a relapse/recurrence-defining event (such as a suicide attempt or referral to a psychiatrist), while $12.6 \%$ did not re-initiate therapy but experienced a relapse/recurrence-defining event. The pattern of use was not independent of relapse/recurrence subtype (Table 3); those in the early discontinuation cohort were disproportionately likely to be coded as relapse/recurrence owing to re-initiation of therapy following a 6-month gap without any antidepressant prescriptions.

The stable use cohort experienced the lowest unadjusted rate of relapse or recurrence; $20 \%$ of the stable users had a relapse or recurrence (Table 2). Higher percentages of patients in the $<120$ days, switching/augmentation and upward titration cohorts experienced a relapse or recurrence $(23 \%, 29 \%$ and $24 \%$, respectively). Univariately, younger age, prescription of an anxiolytic during the prior period and higher number of nonpsychiatric diagnoses in the prior period were significantly associated with increased relapse or recurrence. The Kaplan-Meier curve for time to relapse or recurrence by prescribing pattern is presented in Fig. 1. The survival curves begin to separate within two months following the end of the treatment period.

Results from logistic regression on the risk of a relapse or recurrence are presented in Table 4. The covariate-adjusted OR for patients in the $<120$ days cohort relative to the stable use cohort was 1.16 $(P=0.04)$. Statistically significantly elevated risk of relapse or recurrence was also found for the switching/augmentation cohort $(\mathrm{OR}=1.59, P<0.01)$, but not for the upward titration cohort. The analysis was repeated for each distinct subtype of relapse and recurrence (re-initiation of therapy, event, both). This exercise revealed no major differences in the associations between prescribing pattern and outcome (data not shown).

In this multi-covariate logistic regression model, other factors that independently and significantly influenced the likelihood of experiencing a relapse or recurrence of depression included use in the prior period of anxiolytic medications (OR 2.25, $P<0.001$ ), more comorbid conditions in the prior period (OR 1.07, $P<0.001$ for each additional comorbidity) and age (OR $0.99, P<0.001$ for each oneyear increase in age). 


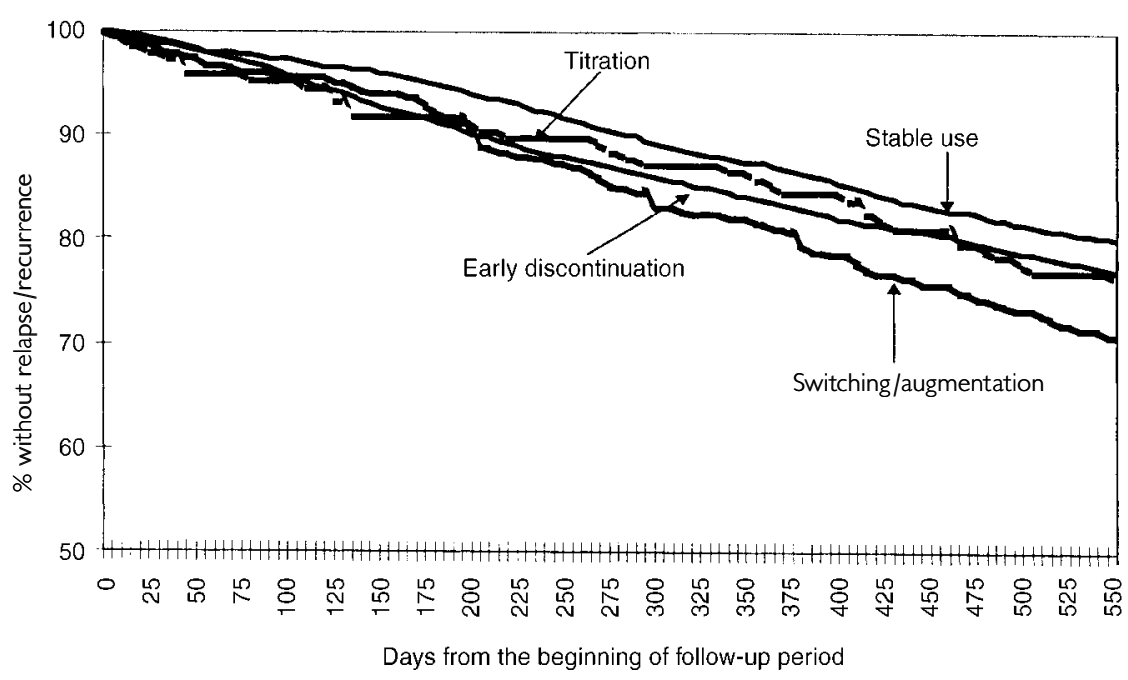

Fig. I Kaplan-Meier curve of time to relapse or recurrence from beginning of follow-up period by prescribing pattern.

Table 4 Logistic regression model: impact of prescribing patterns and other variables on relapse or recurrence in adult patients prescribed an SSRI for depression

\begin{tabular}{|c|c|c|}
\hline & OR & $95 \% \mathrm{Cl}$ \\
\hline \multicolumn{3}{|l|}{ Prescription pattern } \\
\hline$<120$ days & 1.16 & $1.01-1.33$ \\
\hline Switching/augmentation & 1.59 & $1.19-2.11$ \\
\hline Upward titration & 1.20 & $0.79-1.77$ \\
\hline Stable use & 1.00 & - \\
\hline \multicolumn{3}{|l|}{ Comorbidity } \\
\hline Anxiolytic use in prior period & 2.25 & $1.44-3.47$ \\
\hline No anxiolytic use in prior period & 1.00 & - \\
\hline Number of prior-period comorbidities & 1.07 & I.03-I.II \\
\hline \multicolumn{3}{|l|}{ Index year } \\
\hline 1993 & $\mathrm{I} .14$ & $1.00-1.31$ \\
\hline 1994 & 1.14 & $1.00-1.30$ \\
\hline 1995 & 1.00 & - \\
\hline \multicolumn{3}{|l|}{ Patient characteristics } \\
\hline Age & 0.99 & $0.99-1.00$ \\
\hline Female & 1.05 & $0.93-1.20$ \\
\hline Male & 1.00 & - \\
\hline Cohabiting & 1.07 & $0.57-1.89$ \\
\hline Divorced & 1.03 & $0.70-1.47$ \\
\hline Separated & 1.10 & $0.55-2.07$ \\
\hline Single & 1.12 & $0.94-1.33$ \\
\hline Widowed & 0.93 & $0.60-1.39$ \\
\hline Married or unknown & 1.00 & - \\
\hline Region I' & 0.95 & $0.8 \mathrm{I}-\mathrm{I} . \mathrm{II}$ \\
\hline Region 2 & 1.09 & $0.93-1.27$ \\
\hline Region 3 & 1.02 & $0.88-1.17$ \\
\hline Region 4 & 1.00 & - \\
\hline
\end{tabular}

SSRI, selective serotonin reuptake inhibitor.

I. Regions are defined in Table 2.

\section{DISCUSSION}

Among patients treated for depression with an SSRI, those who remained on their initial SSRI for at least 120 days experienced the lowest risk of relapse or recurrence of depression. Those who discontinued their antidepressant therapy prematurely were at significantly higher risk for relapse or recurrence, as were patients whose initial SSRI was switched or augmented.

\section{Observed prescribing patterns}

As in other studies conducted in clinical practice settings, but contrary to international treatment guidelines, a strikingly larger percentage of patients in this study discontinued treatment less than 120 days after initiation of therapy (Katon et al, 1992; Donoghue \& Tylee, 1996; MacDonald et al, 1996, 1997; Thompson et al, 1996; Quik et al, 1998). Some premature discontinuation of antidepressant therapy may reflect treatment failure, probably reflecting either a physician or patient decision since first-line treatment failures would normally be switched to another antidepressant. Alternatively, a patient may decide that there is no reason to continue treatment after resolution of the initial depressive symptoms, even if recommended to do so by a physician. It is also possible that a small portion of patients did not meet criteria for major depression and thus did not warrant treatment with an antidepressant. However, evidence of depression-related diagnosis was noted in all study subjects, and Donoghue (1996) validated the hypothesis that such 'depression-related diagnoses' could be used as a proxy for the diagnosis of depression. It follows that a more likely reason for discontinuing therapy within 120 days is a lack of patient adherence to the recommended length of treatment or a lack of recognition by general practitioners of the importance of continuation and maintenance therapy in major depressive disorder.

In both the switching/augmentation and upward titration cohorts, the duration of treatment was actually longer than among patients in the stable use cohort, but the risk of relapse or recurrence was significantly higher, suggestive of treatment failure or difficult-to-treat depression. This prescribing pattern may indicate that an optimal response was delayed, incomplete 
or never achieved, elevating the risk of relapse or recurrence. Patients whose SSRI dose was titrated may also have experienced delayed time to optimal response, but risk of relapse or recurrence was not significantly elevated. This finding may be due in part to the small number of patients in this group.

\section{Other risk factors}

Consistent with previous studies, younger age and more non-psychiatric comorbid conditions were significantly associated with higher risk for relapse or recurrence (Keller et al, 1983; Lewinsohn et al, 1989; Keller, 1992; Ramana et al, 1995). However, care should be taken in the interpretation of this strong explanatory effect of comorbid conditions in predicting a relapse or recurrence of depression because the definition of relapse or recurrence in this study is based on health-care resource use. Persons having a larger number of comorbid conditions may seek health care more frequently and are therefore more likely to be recognised and treated for relapse or recurrence. Furthermore, comorbid conditions may be the somatic manifestation of depression in some patients, thereby serving as a proxy measure of depression severity. Another significant predictor of relapse and recurrence was use of an anxiolytic agent in the prior period. This may reflect undiagnosed depression with anxiety during the prior period, and hence more severe depression at the index date, consistent with a higher risk of relapse or recurrence.

\section{Sample considerations}

The strength of this analysis is the naturalistic character of the data, despite limitations inherent in any records-based analysis. The patterns and associations observed here are a true reflection of prescribing patterns and their sequelae in primary care in the UK. We included only patients who initiated treatment with an SSRI, in order to elucidate more clearly the role of the prescribing patterns themselves. By restricting the sample to SSRIs, a source of variation was significantly reduced. Although tricyclic antidepressants (TCAs) have been shown to have similar efficacy to SSRIs in clinical trials, the sideeffect profile or other characteristics of TCAs may lead to a higher rate of early discontinuation of treatment relative to SSRIs, as observed both in clinical trials
(Montgomery et al, 1994; Anderson \& Tomenson, 1995) and in clinical practice (Katon et al, 1992; Simon et al, 1996; Croghan et al, 1997). The focus on SSRIs is also relevant given their increasing use as first-line agents in the treatment of depression in the UK and around the world.

\section{Effect size limitation}

Although the differences in risk observed for the various prescribing patterns were statistically significant, the effect size is small. This naturally raises the question of clinical relevance $v$. statistical significance. In at least one study in a primary care setting, improvement in drug doses and treatment length resulted in measurable clinical end-point improvements among those with major depression (Katon et al, 1995). However, a post hoc analysis suggested that this effect was driven primarily by those who required a medication change during treatment, suggesting that interventions to increase adherence to treatment should perhaps be more carefully targeted at appropriate patient groups. Therefore, although an effect is seen in the present study, some caution is warranted in the interpretation.

Alternatively, the results here may actually underestimate the effect size. Our retrospective clinical practice data were completely unconstrained by rigorous inclusion or exclusion criteria as in a more traditional randomised clinical trial or a prospective naturalistic study. The resulting heterogeneous population, with regard to diagnosis criteria and severity of depression, may markedly dilute or attenuate observable differences in risk associated with the various patterns of use. Furthermore, the definition used here is inherently skewed towards recurrence: the indicators of relapse or recurrence had to occur a minimum of 6 months after diagnosis and initial treatment. The definition of relapse or recurrence is quite specific, but not very sensitive. Relapse or recurrence of depression is thus potentially significantly underestimated, resulting in a further dilution of risk ratios.

\section{Limitations of definition}

Another limitation is the use of resourceutilisation-based indicators to identify the relapse and recurrence of depression. Such measures are not as reliable or consistent as more formal clinical rating scales, but such scales are not routinely used or recorded in a typical primary care practice. Additionally, there is heterogeneity within the definition of relapse or recurrence. As expected, the type of relapse or recurrence was not independent of the prescribing pattern. It is also noteworthy that those who discontinued early had more opportunity to re-initiate therapy. However, re-initiation of therapy after 6 months or more with no antidepressant therapy is not likely to constitute continuation of the treatment of the original episode, regardless of the duration of treatment for the original episode. Likewise, an event such as admission to hospital or a suicide attempt is indicative of a worsening of depression that rises to the level of relapse or recurrence (whether the patient is on medication or not). Therefore, although the type of relapse or recurrence event was skewed by prescription pattern, the subtypes are each clinically relevant markers of relapse or recurrence. Furthermore, repetition of the analysis by relapse or recurrence subtype revealed no major differences in results. Taken together, these findings reinforce the notion that the three subtypes although differentially distributed by use pattern - captured the same clinical phenomena of relapse or recurrence.

\section{REFERENCES}

Anderson, I. M. \& Tomenson, B. M. (1995) Treatment discontinuation with selective serotonin reuptake inhibitors compared with tricyclic antidepressants: a meta-analysis. British Medical Journal, 310, 1433-1438.

Chisholm, J. (1990) The Read clinical classification. British Medical Journal, 300, 1092.

Croghan, T. W., Lair, T. J., Engelhart, L., et al (1997) Effect of antidepressant therapy on health care utilization and costs in primary care. Psychiatric Services, 48, $1420-1426$

Depression Guideline Panel (1993) Depression in Primary Care: Vol. 2. Treatment of Major Depression. Clinical Practice Guideline, No. 5 (Agency for Health Care Policy and Research publication No. 93-0550). Rockville, MD: US Department of Health and Human Service.

Donoghue, J. M. (1996) Prescribing patterns of selective serotonin reuptake inhibitors in primary care: a naturalistic follow-up study. Journal of Serotonin Research, 4, 267-270.

— \& Tylee, A. (1996) The treatment of depression: prescribing patterns of antidepressants in primary care in the UK. British Journal of Psychiatry, 168, 164-168.

Frank, E., Kupfer, D. J., Perel, J. M., et al (1990)

Three-year outcomes for maintenance therapies in recurrent depression. Archives of General Psychiatry, 47, 1093-1099.

Katon, W., von Korff, M., Lin, E., et al (1992) Adequacy and duration of antidepressant treatment in primary care. Medical Care, 30, 67-76. 
_ , _ , _ , et al (1995) Collaborative management to achieve treatment guidelines: impact on depression in primary care. Journal of the American Medical Association, 273, $1026-103 \mid$

Keller, M. B. (1992) The naturalistic course of anxiety and depressive disorders. Clinical Neuropharmacology, $\mathbf{1 5}$ (suppl. I, part A), 17I-173.

_ , Lavori, P. W., Lewis, C. E., et al (1983) Predictors of relapse in major depressive disorder. Journal of the American Medical Association, 250, 3299-3304.

Kocsis, J. H., Friedman, R. A. \& Markowitz, J. C. (1996) Maintenance therapy for chronic depression: a controlled clinical trial of desipramine. Archives of General Psychiatry, 53, 769-774.

\section{Lewinsohn, P. M., Zeiss, A. M. \& Duncan, E. M.} (1989) Probability of relapse after recovery from an episode of depression. Journal of Abnormal Psychology, 98 $107-116$

\section{MacDonald, T., McMahon, A. D. \& Reid, I. C. (1996)} Antidepressant drug use in primary care: a record linkage study in Tayside, Scotland. British Medical Journal, 313, 860-86.

_, Reid, I. C. \& McMahon, A. D. (1997) Patients receive an inadequate dose of antidepressants for an inadequate period (letter, comment). British Medical Journal, 315, 56.

Maj, M., Veltro, F., Pirozzi, R., et al (1992) Pattern of recurrence of illness after recovery from an episode of major depression: a prospective study. American Journal of Psychiatry, 149, 795-800.

Melfi, C. A., Chawla, A. J., Croghan, T. W., et al (1998)

The effects of adherence to antidepressant treatment guidelines on relapse or recurrence of depression. Archives of General Psychiatry, 55, 1128-1132.

Montgomery, S. A., Bebbington, P., Cowen, P., et al (1993) Guidelines for treating depressive illness with antidepressants. Journal of Psychopharmacology, 7, 19-23.

\section{_ , Henry, J., McDonald, G., et al (1994) Selective}

serotonin reuptake inhibitors: meta-analysis of

discontinuation rates. International Clinical

Psychopharmacology, 9, 47-53.

Old Age Depression Interest Group (1993) How long should the elderly take antidepressants? A double-blind placebo-controlled study of continuation/prophylaxis therapy with dothiepin. British Journal of Psychiatry, 162 $175-182$

Paykel, E. S. \& Priest, R. G. (1992) Recognition and management of depression in general practice: consensus statement. British Medical Journal, 305 |198-1202.

Prien, R. F. \& Kupfer, D. J. (1986) Continuation drug therapy for major depressive episodes: how long should

\section{CLINICAL IMPLICATIONS}

Early discontinuation of antidepressant therapy for depression may increase the risk of relapse or recurrence.

Selective serotonin reuptake inhibitors (SSRIs) should be prescribed for depression at the therapeutic dose and patients should be encouraged to adhere to long-term treatment consistent with international treatment guidelines.

Among patients treated for depression with SSRIs, those with prior anxiolytic use, more comorbid conditions or younger age should be monitored carefully in order to prevent relapse or recurrence of depression.

\section{LIMITATIONS}

- The effect size observed for alternative prescribing patterns is small.

- A utilisation-based definition of relapse or recurrence was used.

- Relapse was not distinguishable from recurrence in this study.

AMI J. CLAXTON, PhD, Global Health Outcomes Research, Eli Lilly and Company, Indianapolis, IN and Indiana University School of Medicine, Department of Public Health, Indianapolis, IN; ZHIMING LI, PhD, Pharmacia \& Upjohn, Kalamazoo, MI; JAN McKENDRICK, MSc, Health Outcomes, Eli Lilly and Company Limited, Basingstoke, UK

Correspondence: Dr Ami J. Claxton, Global Health Outcomes Research, Eli Lilly and Company, Indianapolis, IN 46285, USA. Tel: 317-277-3494; Fax: 317-276-6026; e-mail: ami.claxton@lilly.com

(First received 14 June 1999, final revision 20 December 1999, accepted 21 December 1999)

it be maintained? American Journal of Psychiatry, 143, $18-23$

Quik, R. F. P., Kleintjens, H. \& Hylan, T. R. (1998)

Prescriptiepatronen van antidepressiva in de

huisartspraktijk en hun invloed op het gebruik van zorgvoorzieningen. Het Pharmaceutisch Weekblad, 133 1078-1085.

Ramana, R., Paykel, E. S., Cooper, Z., et al (1995) Remission and relapse in major depression: a two-year prospective follow-up study. Psychological Medicine, 25. $|16|-1170$.
Simon, G. E., Von Korff, M., Heiligenstein, J. H., et al (1996) Initial antidepressant choice in primary care: effectiveness and cost of fluoxetine vs tricyclic antidepressants. Journal of the American Medical Association, 275, 1897-1902.

Thompson, D., Buesching, D., Gregor, K. J., et a (1996) Patterns of antidepressant use and their relation to costs of care. American Journal of Managed Care, $\mathbf{2}$, 1239-1246.

World Health Organization (1978) The Ninth Revision of the International Classification of Diseases and Related Health Problems (ICD-9). Geneva: WHO. 